Washington University School of Medicine Digital Commons@Becker

Open Access Publications

2019

\title{
Trial of SAGE-217 in patients with major depressive disorder
}

\author{
Handan Gunduz-Bruce \\ Sage Therapeutics \\ Christopher Silber \\ Sage Therapeutics \\ Inder Kaul \\ Kaul Consulting \\ Anthony J. Rothschild \\ University of Massachusetts Medical School \\ Robert Riesenberg \\ The Atlanta Center for Medical Research
}

See next page for additional authors

Follow this and additional works at: https://digitalcommons.wustl.edu/open_access_pubs

Please let us know how this document benefits you.

\section{Recommended Citation}

Gunduz-Bruce, Handan; Silber, Christopher; Kaul, Inder; Rothschild, Anthony J.; Riesenberg, Robert; Sankoh, Abdul J.; Li, Haihong; Lasser, Robert; Zorumski, Charles F.; Rubinow, David R.; Paul, Steven M.; Jonas, Jeffrey; Doherty, James J.; and Kanes, Stephen J., "Trial of SAGE-217 in patients with major depressive disorder." The New England Journal of Medicine. 381, 10. 903 - 911. (2019).

https://digitalcommons.wustl.edu/open_access_pubs/8122

This Open Access Publication is brought to you for free and open access by Digital Commons@Becker. It has been accepted for inclusion in Open Access Publications by an authorized administrator of Digital Commons@Becker. For more information, please contact vanam@wustl.edu. 


\section{Authors}

Handan Gunduz-Bruce, Christopher Silber, Inder Kaul, Anthony J. Rothschild, Robert Riesenberg, Abdul J. Sankoh, Haihong Li, Robert Lasser, Charles F. Zorumski, David R. Rubinow, Steven M. Paul, Jeffrey Jonas, James J. Doherty, and Stephen J. Kanes 


\title{
The NEW ENGLAN D \\ JOURNAL of MEDICINE
}

ESTABLISHED IN 1812

SEPTEMBER 5, 2019

VOL. 381 NO. 10

\section{Trial of SAGE-217 in Patients with Major Depressive Disorder}

\author{
Handan Gunduz-Bruce, M.D., Christopher Silber, M.D., Inder Kaul, M.D., Anthony J. Rothschild, M.D., \\ Robert Riesenberg, M.D., Abdul J. Sankoh, Ph.D., Haihong Li, Ph.D., Robert Lasser, M.D., \\ Charles F. Zorumski, M.D., David R. Rubinow, M.D., Steven M. Paul, M.D., Jeffrey Jonas, M.D., \\ James J. Doherty, Ph.D., and Stephen J. Kanes, M.D., Ph.D.
}

\section{ABSTRACT}

\section{BACKGROUND}

Altered neurotransmission of $\gamma$-aminobutyric acid (GABA) has been implicated in the pathogenesis of depression. Whether SAGE-217, an oral, positive allosteric modulator of GABA type A receptors, is effective and safe for the treatment of major depressive disorder is unknown.

\section{METHODS}

In this double-blind, phase 2 trial, we enrolled patients with major depression and randomly assigned them in a 1:1 ratio to receive $30 \mathrm{mg}$ of SAGE-217 or placebo once daily. The primary end point was the change from baseline to day 15 in the score on the 17-item Hamilton Depression Rating Scale (HAM-D; scores range from 0 to 52, with higher scores indicating more severe depression). Secondary efficacy end points, which were assessed on days 2 through 8 and on days 15, 21, 28, 35, and 42, included changes from baseline in scores on additional depression and anxiety scales, a reduction from baseline of more than $50 \%$ in the HAM-D score, a HAM-D score of 7 or lower, and a Clinical Global Impression of Improvement score of 1 (very much improved) or 2 (much improved) (on a scale of 1 to 7 , with a score of 7 indicating that symptoms are very much worse).

\section{RESULTS}

A total of 89 patients underwent randomization: 45 patients were assigned to the SAGE217 group, and 44 to the placebo group. The mean baseline HAM-D score was 25.2 in the SAGE-217 group and 25.7 in the placebo group. The least-squares mean $( \pm S E)$ change in the HAM-D score from baseline to day 15 was $-17.4 \pm 1.3$ points in the SAGE-217 group and $-10.3 \pm 1.3$ points in the placebo group (least-squares mean difference in change, -7.0 points; $95 \%$ confidence interval, -10.2 to $-3.9 ; \mathrm{P}<0.001)$. The differences in secondary end points were generally in the same direction as those of the primary end point. There were no serious adverse events. The most common adverse events in the SAGE-217 group were headache, dizziness, nausea, and somnolence.

\section{CONCLUSIONS}

Administration of SAGE-217 daily for 14 days resulted in a reduction in depressive symptoms at day 15. Adverse events were more common in the SAGE-217 group than in the placebo group. Further trials are needed to determine the durability and safety of SAGE217 in major depressive disorder and to compare SAGE-217 with available treatments. (Funded by Sage Therapeutics; ClinicalTrials.gov number, NCT03000530.)
From Sage Therapeutics, Cambridge (H.G.-B., C.S., A.J.S., H.L., R.L., S.M.P., J.J., J.J.D., S.J.K.), Kaul Consulting, Concord (I.K.), and the University of Massachusetts Medical School and UMass Memorial Healthcare, Worcester (A.J.R.) - all in Massachusetts; the Atlanta Center for Medical Research, Atlanta (R.R.); Washington University School of Medicine, St. Louis (C.F.Z., S.M.P.); and the University of North Carolina School of Medicine, Chapel Hill (D.R.R.). Address reprint requests to Dr. Gunduz-Bruce at Sage Therapeutics, 215 First St., Cambridge, MA 02142, or at handan.gunduz-bruce@sagerx.com.

N Engl J Med 2019;381:903-11. DOI: 10.1056/NEJMoa1815981

Copyright (c) 2019 Massachusetts Medical Society 
A Quick Take is available at NEJM.org

\section{A}

NTIDEPRESSANTS THAT PRIMARILY ENhance monoaminergic neurotransmission involving serotonin or norepinephrine are used in the treatment of major depressive disorder, and their clinical effects are generally evident in 4 to 8 weeks. ${ }^{1-3}$ One hypothesis for the mechanism of depression implicates deficits in $\gamma$-aminobutyric acid (GABA) and downstream alterations in monoaminergic neurotransmission. ${ }^{4}$ This hypothesis is supported by evidence that reduced GABA levels have been observed in plasma, cerebrospinal fluid, ${ }^{5-7}$ and cortical brain tissues $^{8-11}$ of patients with depression. In addition, reduced expression of GABA-synthesizing enzymes in the brain tissue of persons who have died by suicide, ${ }^{12,13}$ a reduced number of GABAergic interneurons in the brain tissue of patients with depression, ${ }^{14}$ and reduced mRNA for GABA type A $\left(\mathrm{GABA}_{\mathrm{A}}\right) \alpha 4$ and $\delta$ subunits (which encode extrasynaptic GABA $\mathrm{A}_{\mathrm{A}}$ receptors) in the brain tissue of persons with depression who have died by suicide ${ }^{15}$ have been observed.

Neurosteroids, which are synthesized from cholesterol in the brain, are potent modulators of GABA and glutamate. ${ }^{16}$ Despite their steroid structure, their target activity and pharmacologic characteristics are distinct from those of glucocorticoids and differ in both genomic and nongenomic effects. ${ }^{17,18}$ Preclinical studies have shown that the naturally occurring neurosteroid allopregnanolone is a positive allosteric modulator of synaptic and extrasynaptic $\mathrm{GABA}_{\mathrm{A}}$ receptors that affects both phasic and tonic inhibition of neurons. ${ }^{16,19}$ In rodents, allopregnanolone is synthesized locally in the brain in response to acute stressors. ${ }^{19-21}$ With experimental chronic stress, however, levels of allopregnanolone in the brain decrease, and behavioral changes normalize after treatments that increase allopregnanolone levels. ${ }^{19,22,23}$

Reduced levels of allopregnanolone in the cerebrospinal fluid normalize after successful treatment of depression with antidepressants. ${ }^{24}$ Placebo-controlled trials in patients with postpartum depression have shown efficacy of a 60hour infusion of brexanolone, an intravenous formulation of allopregnanolone, which supports the hypothesis of GABAergic dysfunction in that form of depression. ${ }^{25,26}$

SAGE-217 - an oral, synthetic neurosteroid and positive allosteric modulator of $\mathrm{GABA}_{\mathrm{A}}$ receptors - has shown anticonvulsant, anxiolytic, and sedative properties in studies of seizure in rodent models. ${ }^{27-29}$ A functional assay measuring both agonistic and antagonistic effects of SAGE217 has indicated no activity on a panel of 22 nuclear hormone receptors. ${ }^{30}$

Studies of single ascending and multiple ascending doses in healthy volunteers have shown that SAGE-217 has a plasma half-life of 16 to 23 hours, which is compatible with once-daily dosing; sedation is the most common adverse event. ${ }^{31,32}$ We previously conducted an open-label, uncontrolled pilot trial of SAGE-217 involving patients with major depressive disorder who were treated for 14 days. The results suggested that SAGE-217, like intravenous brexanolone (with which it shares a similar molecular pharmacology profile), may be associated with a rapid onset of action. ${ }^{33}$ Here we report the results of a randomized, double-blind, placebo-controlled trial of SAGE-217 in patients with major depressive disorder. The protocol of the current trial (which also contains the protocol of the open-label pilot trial) and the statistical analysis plan are available with the full text of this article at NEJM.org. The results of the pilot trial are provided in the Supplementary Appendix, available at NEJM.org. The pilot trial and the current placebo-controlled trial recruited different patients.

\section{METHODS}

\section{TRIAL DESIGN AND OVERSIGHT}

This phase 2 trial was conducted at eight sites in the United States from April 2017 through October 2017. A list of sites and principal investigators is provided in the Supplementary Appendix. Approval was obtained from the institutional review board at each site, and written informed consent was obtained from each patient. Sage Therapeutics designed the trial, provided the SAGE-217 and placebo, collected and analyzed the data, and paid for professional writing assistance. The qualification process for end-point raters and administration of assessments were overseen by Bracket Global (Wayne, PA) (details are provided in the Supplementary Appendix). Confidentiality agreements exist between the authors and Sage Therapeutics. All the authors vouch for the accuracy and completeness of the data and analyses, the fidelity of the trial to the protocol, and the completeness of reporting of adverse events. 
We recruited patients from outpatient clinics and by using advertising materials approved by the institutional review boards. Patients were admitted as inpatients for the first week of the trial to facilitate daily assessments. Randomization was performed with the use of interactiveresponse technology created by 4G Clinical (Wellesley, MA). Patients were assigned, in a 1:1 ratio, to receive either SAGE-217 (30 mg) or placebo. SAGE-217 or placebo was administered at 8 p.m. with food on days 1 through 14 . Patients who had adverse effects or reported sedation with the $30-\mathrm{mg}$ dose could receive $20 \mathrm{mg}$ daily; patients who had adverse effects or reported sedation with the 20-mg dose could be withdrawn from the trial. Initiation of new antidepressants was not allowed for the duration of the 14-day treatment period. During the 4 weeks after the treatment period, primary psychiatrists or investigators could adjust the dose of existing antidepressants or prescribe new antidepressants for patients as appropriate. No formal psychotherapy was used as part of the trial, except in the case of three patients (two patients in the SAGE-217 group and one patient in the placebo group) who had been receiving psychotherapy for longer than 1 year at trial entry and continued this treatment throughout the trial. Patients had to discontinue the trial regimen if they had a new condition that would have met exclusion criteria, was clinically relevant, or was unsafe (i.e., treatment for the condition was no longer associated with a favorable risk-benefit ratio as judged by the investigator or sponsor), or if they had unacceptable adverse events.

\section{TRIAL PARTICIPANTS}

The trial population included men and women, 18 to 65 years of age, who had received a diagnosis of major depressive disorder and had a score on the 17-item Hamilton Depression Rating Scale (HAM-D) of 22 or higher, which corresponds to moderate-to-severe depression (scores range from 0 to 52, with higher scores indicating more severe depression). ${ }^{34}$ The diagnosis of depression was made with the use of the Structured Clinical Interview for Diagnostic and Statistical Manual of Mental Disorders, fifth edition, Axis I disorders. ${ }^{35}$

Patients were eligible to participate if they had been receiving stable doses of antidepressants for at least 30 days or if they were not taking antidepressants. Key exclusion criteria were a history of a suicide attempt, a history of treatment-resistant depression, a recent history or current clinically significant manifestations of other acute or chronic medical conditions, a positive pregnancy test, a history of seizures, and a history of bipolar disorder, schizophrenia, or schizoaffective disorder. A full list of inclusion and exclusion criteria is provided in the Supplementary Appendix.

\section{END POINTS}

The primary end point was the change in the HAM-D score from baseline to day 15 . The secondary end points, which were assessed on days 2 through 8 and on days 15, 21, 28, 35, and 42, included the change from baseline in HAM-D score; a reduction of more than $50 \%$ from baseline in the HAM-D score; a HAM-D score of 7 or lower; the change from baseline in the score on the Montgomery-Åsberg Depression Rating Scale (MADRS; scores range from 0 to 60, with higher scores indicating more severe depression); the change from baseline in the score on Bech-6 (a subscale of HAM-D that assesses the overall severity of depression on the basis of ratings in six categories [depressed mood, feelings of guilt, work and activities, psychomotor retardation, psychic anxiety, and general somatic symptoms]; scores range from 0 to 100 , with higher scores indicating more severe depression) ${ }^{36}$; the change from baseline in the score on the Hamilton Anxiety Rating Scale (HAM-A; scores range from 0 to 56 , with higher scores indicating more severe anxiety); and a score on the Clinical Global Impression of Improvement (CGI-I) scale of 1 (very much improved) or 2 (much improved) (scores range from 1 to 7, with a score of 7 indicating that symptoms are very much worse). ${ }^{37}$

Safety was assessed on the basis of the frequency and severity of adverse events, vital signs, changes in clinical laboratory measurements, physical examinations, electrocardiography, the Stanford Sleepiness Scale, and assessment of suicidal ideation and behavior with the use of the Columbia Suicide Severity Rating Scale. Adverse events during the treatment period were defined as any adverse events or any worsening of a preexisting medical condition that occurred from the time of administration of the first dose of SAGE-217 or placebo until 7 days after the last dose. 


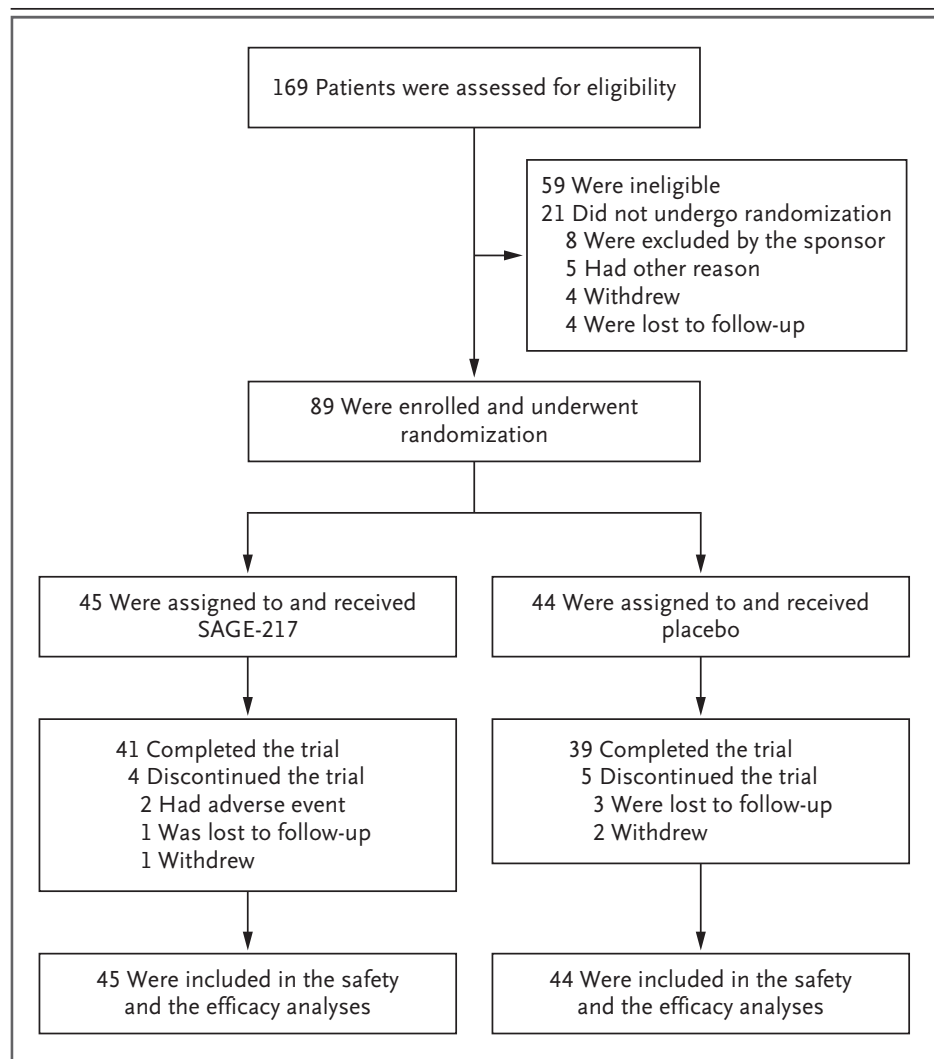

Figure 1. Screening, Randomization, and Follow-up.

\section{STATISTICAL ANALYSIS}

Analyses were performed according to the intention-to-treat principle and included all patients who underwent randomization. Continuous variables are reported as means and standard deviations, and categorical variables as numbers and percentages. The least-squares mean changes from baseline in HAM-D, Bech-6, MADRS, and HAM-A scores were analyzed with the use of mixed-effects models for repeated measures, with treatment, visit, and treatment-by-visit interaction as fixed effects and with adjustment for baseline score, use of antidepressants, and trial site. Categorical measures (i.e., $>50 \%$ reduction in HAM-D score from baseline, HAM-D score $\leq 7$, and CGI-I score of 1 or 2) were analyzed with the use of a generalized estimating equation model, with treatment, visit, and treatment-by-visit interaction as fixed effects and with adjustment for baseline score, use of antidepressants, and trial site. Statistical analyses were performed with the use of SAS software, version 9.3 (SAS Institute). There was no plan for imputation of missing data, but sensitivity analyses were performed to assess the effect of missing data. The statistical analysis plan did not include a provision for correction for multiplicity when the analyses of secondary end points were performed. Therefore, the results

\begin{tabular}{|c|c|c|}
\hline Characteristic & SAGE-217 Group ( $N=45$ ) & Placebo Group ( $N=44)$ \\
\hline Age $-y r$ & $49.1 \pm 13.6$ & $38.3 \pm 12.2$ \\
\hline Male sex — no. (\%) & $20(44)$ & $14(32)$ \\
\hline \multicolumn{3}{|l|}{ Race or ethnic group — no. (\%) † } \\
\hline American Indian or Alaska Native & 0 & 0 \\
\hline Asian & $1(2)$ & 0 \\
\hline Black & $36(80)$ & $28(64)$ \\
\hline Native Hawaiian or Pacific Islander & 0 & 0 \\
\hline White & $7(16)$ & $16(36)$ \\
\hline Other & $1(2)$ & 0 \\
\hline Body-mass indext & $30.0 \pm 6.3$ & $29.9 \pm 5.2$ \\
\hline Baseline HAM-D score $\mathbb{}$ & $25.2 \pm 2.6$ & $25.7 \pm 2.4$ \\
\hline Use of antidepressants at baseline - no. (\%) & $12(27)$ & $10(23)$ \\
\hline Median duration of depression (IQR) — days & $97(63-161)$ & $146(86-204)$ \\
\hline \multicolumn{3}{|c|}{$\begin{array}{l}\text { Plus-minus values are means } \pm S D \text {. IQR denotes interquartile range. } \\
\text { Race was reported by the patients. } \\
\text { The body-mass index is the weight in kilograms divided by the square of the height in meters. } \\
\text { Scores on the 17-item Hamilton Depression Rating Scale (HAM-D) range from } 0 \text { to } 52 \text {, with higher scores indicating more } \\
\text { severe depression. }\end{array}$} \\
\hline
\end{tabular}


are reported as point estimates and unadjusted $95 \%$ confidence intervals and should not be used to infer treatment effects.

\section{RESULTS}

\section{PATIENT CHARACTERISTICS}

A total of 169 patients were screened, of whom 89 were enrolled and underwent randomization. Patients were assigned in a 1:1 ratio to receive SAGE-217 (45 patients) or placebo (44 patients) (Fig. 1). Demographic characteristics were similar in the two groups except that patients in the SAGE-217 group were older than those in the placebo group (mean age, 49 years vs. 38 years), there were fewer women in the SAGE-217 group than in the placebo group ( $56 \%$ vs. $68 \%$ ), and there were more black patients in the SAGE-217 group than in the placebo group (80\% vs. $64 \%$ ) (Table 1). Most patients $(96 \%$ in the SAGE-217 group and $91 \%$ in the placebo group) reported previous depressive episodes. The percentage of patients who were receiving antidepressants at baseline was $27 \%$ in the SAGE-217 group and $23 \%$ in the placebo group, and the duration of this treatment before the patients entered the trial was 2 months to more than 48 months (Table S3 in the Supplementary Appendix). In the SAGE-217 group, 2 patients discontinued the trial drug because of adverse events (1 patient after day 6 and 1 patient after day 10). One patient was lost to follow-up after 9 days, and 1 patient withdrew consent after 10 days (neither of these patients had adverse events). In the placebo group, 3 patients were lost to follow-up during the follow-up period, and 2 patients withdrew from the trial (1 patient after 5 days and 1 patient after 6 days).

\section{EFFICACY}

The mean HAM-D score at baseline was 25.2 in the SAGE-217 group and 25.7 in the placebo group. On day 15, the least-squares mean change from baseline in HAM-D score was $-17.4 \pm 1.3$ points in the SAGE-217 group and $-10.3 \pm 1.3$ points in the placebo group (least-squares mean difference in change, $-7.0 ; 95 \%$ confidence interval $[\mathrm{CI}],-10.2$ to -3.9 ; $\mathrm{P}<0.001$ ) (Table 2). Results of the sensitivity analyses for missing data that used multiple imputation or the last-observation-

\begin{tabular}{|c|c|c|c|c|c|c|}
\hline End Point & $\begin{array}{l}\text { SAGE-217 Group } \\
(\mathrm{N}=45)\end{array}$ & $\begin{array}{l}\text { Placebo Group } \\
\qquad(\mathrm{N}=44)\end{array}$ & Difference & Odds Ratio & $95 \% \mathrm{Cl}$ & $P$ Value \\
\hline $\begin{array}{l}\text { Primary end point: change from baseline } \\
\text { in HAM-D score }{ }^{-}\end{array}$ & $-17.4 \pm 1.3$ & $-10.3 \pm 1.3$ & $-7.0 \pm 1.6$ & & -10.2 to -3.9 & $<0.001$ \\
\hline \multicolumn{7}{|l|}{ Secondary end points } \\
\hline $\begin{array}{c}\text { Reduction of }>50 \% \text { from baseline in } \\
\text { HAM-D score - \% of patients }\end{array}$ & 79 & 41 & & 9.6 & 2.9 to 31.6 & \\
\hline HAM-D score $\leq 7-\%$ of patients & 64 & 26 & & 5.3 & 2.1 to 13.3 & \\
\hline Change from baseline in Bech-6 score & $-40.7 \pm 3.3$ & $-25.7 \pm 3.4$ & $-15.1 \pm 4.1$ & & -23.3 to -6.8 & \\
\hline $\begin{array}{l}\text { Change from baseline in MADRS } \\
\text { score } \mathbb{}\end{array}$ & $-22.5 \pm 1.9$ & $-15.0 \pm 1.9$ & $-7.6 \pm 2.4$ & & -12.3 to -2.8 & \\
\hline $\begin{array}{l}\text { Change from baseline in HAM-A } \\
\text { score }\end{array}$ & $-13.2 \pm 1.1$ & $-8.6 \pm 1.1$ & $-4.6 \pm 1.3$ & & -7.3 to -2.0 & \\
\hline CGI-I score of 1 or $2-\%$ of patients\| & 79 & 45 & & 8.6 & 2.5 to 29.5 & \\
\hline
\end{tabular}

* Plus-minus values are least-squares means \pm SE. The least-squares mean changes from baseline in the scores on the HAM-D, Bech-6, Montgomery-Åsberg Depression Rating Scale (MADRS), and Hamilton Anxiety Rating Scale (HAM-A) were calculated with the use of a mixed-effects model for repeated measures. The least-squares mean differences are for SAGE-217 minus placebo. The listed odds ratios were calculated with the use of a generalized estimating equation model, with treatment, visit, and treatment-by-visit interaction as fixed effects and with adjustment for baseline score, use of antidepressants, and trial site. The $95 \%$ confidence intervals for secondary end points have not been adjusted for multiple testing. There was no plan for imputation of missing data for primary or secondary end points.

$\dagger$ Data are missing for 3 patients in the SAGE-217 group and 2 patients in the placebo group.

$\checkmark$ Scores on the Bech- 6 subscale of the HAM-D range from 0 to 100 , with higher values indicating more severe depression.

$\int$ MADRS scores range from 0 to 60 , with higher scores indicating more severe depression.

I HAM-A scores range from 0 to 56 , with higher scores indicating more severe anxiety.

$\|$ A score of 1 on the Clinical Global Impression of Improvement (CGI-I) scale indicates that symptoms are very much improved, and a score of 2 indicates that symptoms are much improved; scores range from 1 to 7 , with a score of 7 indicating that symptoms are very much worse. 


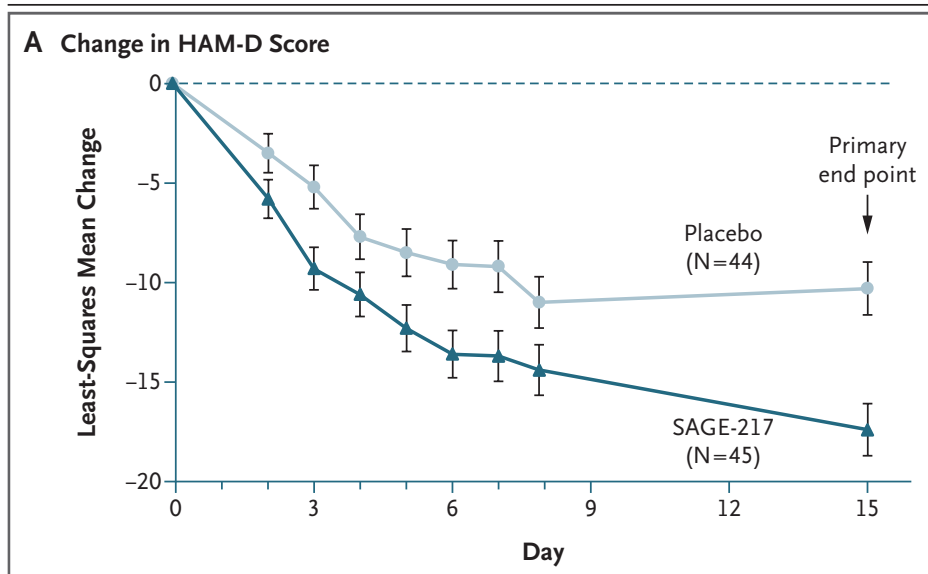

B Reduction of More Than $50 \%$ in HAM-D Score

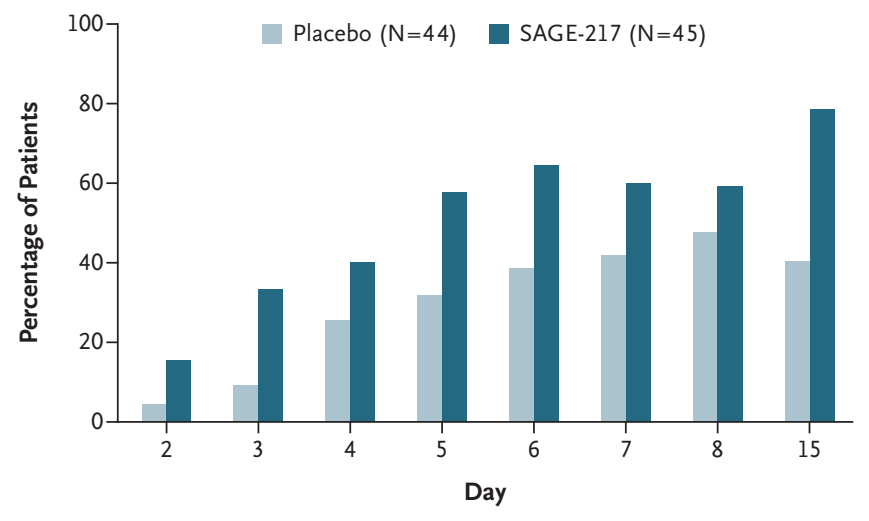

C HAM-D Score of 7 or Lower

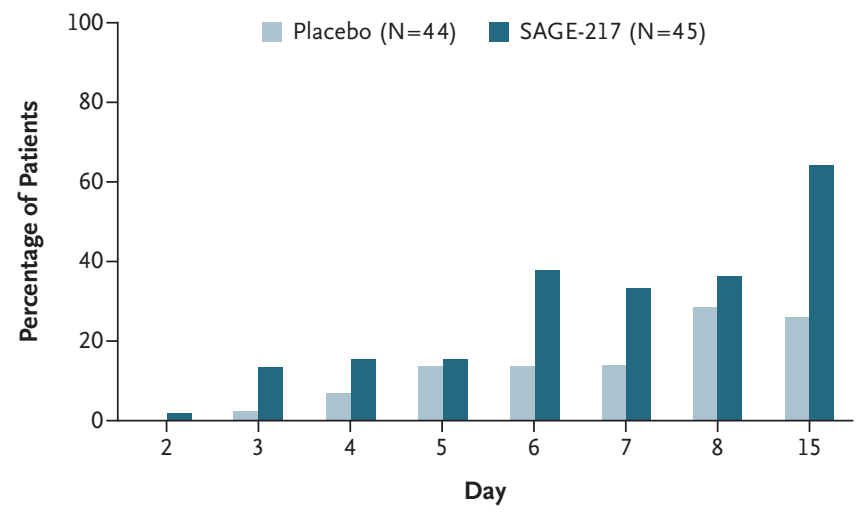

Figure 2. Efficacy End Points.

Scores on the 17-item Hamilton Depression Rating Scale (HAM-D) range from 0 to 52, with higher scores indicating more severe depression. The primary end point was the change in HAM-D score from baseline to day 15 (Panel A). I bars indicate \pm 1 SE. The percentage of patients who had a reduction from baseline in HAM-D score of more than $50 \%$ (Panel B) and the percentage of patients who had a HAM-D score of 7 or lower (Panel C) were secondary end points. carried-forward method were similar to those of the primary analysis (Table S4 in the Supplementary Appendix). At each time point from day 2 through day 28 , the unadjusted $95 \%$ confidence intervals for the between-group difference in the change from baseline in HAM-D scores did not include zero, and the differences were generally in the same direction as those of the primary outcome, but the unadjusted $95 \%$ confidence intervals for days 35 and 42 included zero (Table S5 and Fig. S3A in the Supplementary Appendix). At day 15, the percentage of patients who had a reduction of more than $50 \%$ from baseline in the HAM-D score was $79 \%$ in the SAGE-217 group and $41 \%$ in the placebo group (model-based odds ratio [analyzed with the use of a generalized estimating equation model], 9.6; 95\% CI unadjusted for multiplicity, 2.9 to 31.6); the percentage of patients with a HAM-D score of 7 or lower was $64 \%$ in the SAGE-217 group and $26 \%$ in the placebo group (model-based odds ratio, 5.3; unadjusted 95\% CI, 2.1 to 13.3) (Fig. $2 \mathrm{~B}$ and $2 \mathrm{C}$ and Table 2, and Table $\mathrm{S} 5$ in the Supplementary Appendix). At day 28, the percentage of patients who had a reduction of more than $50 \%$ from baseline in the HAM-D score was $62 \%$ in the SAGE-217 group and $46 \%$ in the placebo group, and the percentage of patients with a HAM-D score of 7 or lower was $52 \%$ and $28 \%$, respectively (Fig. S3B and S3C and Table S5 in the Supplementary Appendix).

During the follow-up period, 3 patients in the SAGE-217 group and 11 patients in the placebo group were given concomitant antidepressants by investigators (Table S7 in the Supplementary Appendix). Patients and investigators remained unaware of the group assignments throughout the follow-up period. Results regarding the effects of SAGE-217 and placebo in patients who received and in patients who did not receive concomitant antidepressants are provided in Table S6 in the Supplementary Appendix.

Results for secondary end points are shown in Table 2. At day 15, the least-squares mean change from baseline in the Bech- 6 score was $-40.7 \pm 3.3$ points in the SAGE-217 group and $-25.7 \pm 3.4$ points in the placebo group (leastsquares mean difference in change, -15.1 points; unadjusted $95 \% \mathrm{CI},-23.3$ to -6.8 ). At day 15 , the least-squares mean change from baseline in the MADRS score was $-22.5 \pm 1.9$ in the SAGE-217 


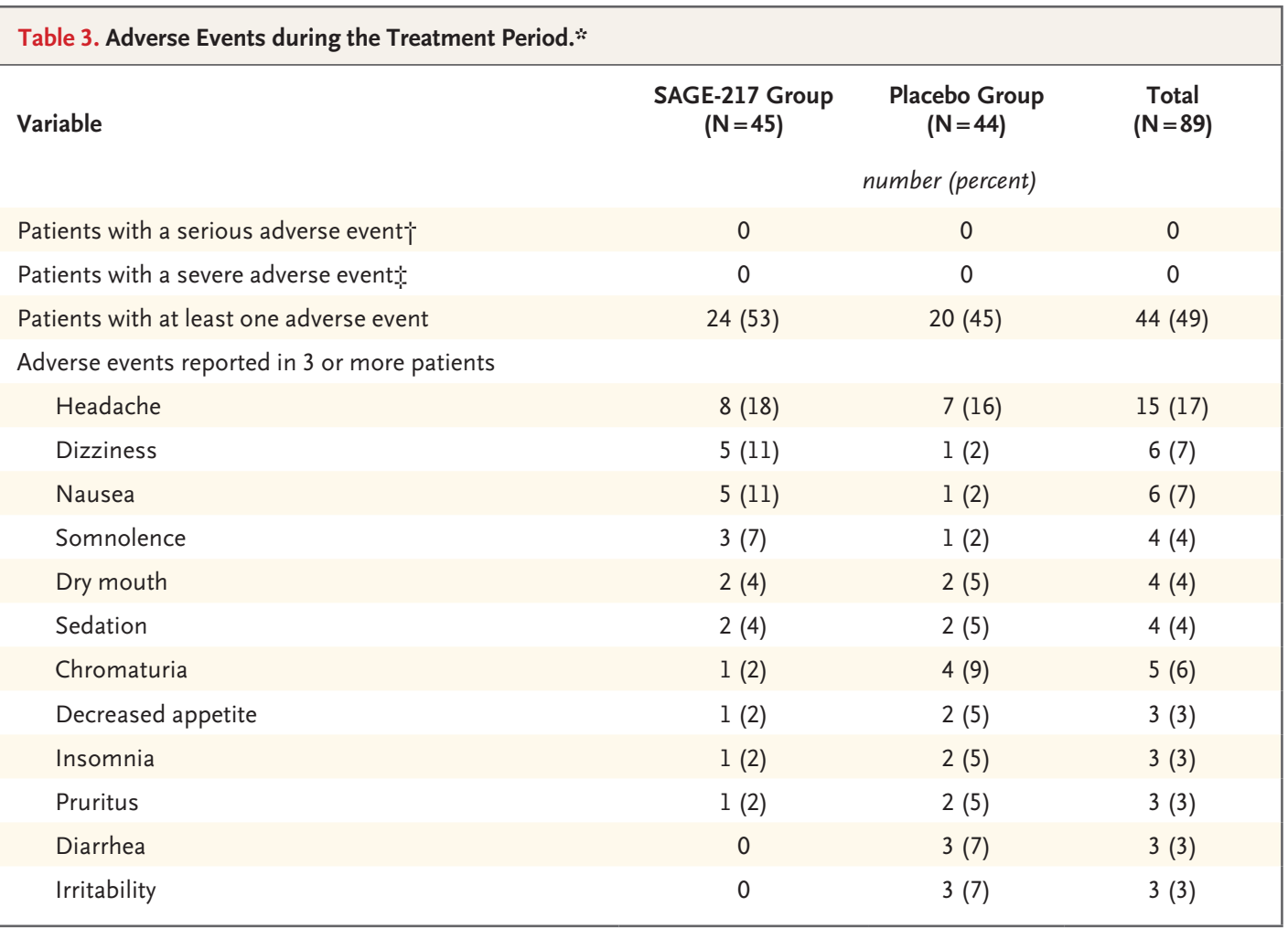

* Adverse events during the treatment period are those that started or worsened from the time of the first dose of the trial intervention through 7 days after the last dose.

$\dagger$ A serious adverse event was defined as any adverse event, occurring while the patient was receiving the trial medication or placebo, that resulted in death, was immediately life-threatening, led to inpatient hospitalization or prolongation of hospitalization, caused persistent or clinically significant disability or incapacity, or resulted in a congenital abnormality or birth defect.

† ties of daily living.

group and $-15.0 \pm 1.9$ in the placebo group (leastsquares mean difference in change, -7.6 points; unadjusted $95 \% \mathrm{CI},-12.3$ to -2.8 ). At all time points, a higher percentage of patients in the SAGE-217 group than in the placebo group had a CGI-I score of 1 or 2 , and the least-squares mean change from baseline in HAM-A score was greater in the SAGE-217 group than in the placebo group, but no inferences can be made because of the lack of adjustment for multiplicity in the analyses of secondary outcomes. (Additional data on secondary end points are provided in Figs. S4 through S6 and Tables S8 through S10 in the Supplementary Appendix.)

\section{SAFETY}

There were no serious adverse events and no deaths during this trial. The percentage of patients who had at least one adverse event during the treatment period was 53\% in the SAGE-217 group and $45 \%$ in the placebo group (Table 3). During days 21 through 42, a total of 4 additional adverse events in the SAGE-217 group and 10 additional adverse events in the placebo group were reported (Table S11 in the Supplementary Appendix). Two patients in the SAGE-217 group discontinued the trial drug because they met a protocol criterion for discontinuation (one patient after day 6 because of nausea, dizziness, and headache, and the other patient after day 10 because of increased levels of alkaline phosphatase, alanine aminotransferase, aspartate aminotransferase, and $\gamma$-glutamyltransferase [this patient had mildly elevated baseline values and was asymptomatic throughout the trial, and all values returned to baseline or near-baseline levels after discontinuation of the trial medication]).

The most common adverse events that oc- 
curred in at least $5 \%$ of patients in the SAGE-217 group were headache, dizziness, nausea, and somnolence (Table 3). Subjective sleepiness, as assessed with the use of the Stanford Sleepiness Scale, was similar in the two groups. The score on the Stanford Sleepiness Scale was assessed before and after administration of SAGE-217 or placebo at multiple time points daily; data for days $1,7,14$, and 15 are summarized in Table S12 in the Supplementary Appendix. In the SAGE-217 group, adverse events occurred in $67 \%$ of patients who received concomitant antidepressants and in $48 \%$ of patients who received SAGE217 as monotherapy (Table S13 in the Supplementary Appendix). Six patients in the SAGE-217 group had dose reductions (from $30 \mathrm{mg}$ to $20 \mathrm{mg}$ ) - five as the result of adverse events (dizziness [in two patients], somnolence [in one patient], sedation [in one patient], and nausea and vomiting [in one patient]) and one because of patientreported sleepiness as assessed by the Stanford Sleepiness Scale. No patients in the placebo group had dose reductions.

\section{DISCUSSION}

Among patients with moderate-to-severe major depressive disorder, treatment with SAGE-217 for 14 days resulted in a reduction in depressive symptoms, as assessed on the basis of the change in the HAM-D score from baseline to day 15 and of changes in secondary end-point assessments that also were generally in the same direction as those of the primary outcome. The trial was not designed to confirm findings beyond the 15-day assessment period. One patient reported euphoria as an adverse event, which suggests that in most patients the effect of SAGE-217 on alleviation of depressive symptoms was not related to a temporary feeling of euphoria. The assessment of scores on the Bech- 6 subscale showed improvement in all core symptoms of depression among patients who received SAGE-217. The effect of SAGE-217 was similar among patients who received adjunctive treatment with conventional antidepressants and those who received SAGE-217 as monotherapy, although the number of patients in these groups was small, and the trial was not powered to assess this comparison. These findings may support a role for $\mathrm{GABA}_{\mathrm{A}}$ receptors in the pathophysiology of depression. ${ }^{4,38}$ On the basis of the pharmacologic properties of SAGE-217 as a positive allosteric modulator of $\mathrm{GABA}_{\mathrm{A}}$ receptors, somnolence and sedation may be expected adverse events, and these were among the most common adverse events in the trial.

Limitations of the current trial include the small sample size, lack of adjustment for multiplicity in the testing of secondary end-point results, limited diversity in racial representation among the participants, and power to detect changes in depression severity only at day 15 and not through the entire follow-up period. Data were missing for approximately $5 \%$ of patients in each group, but sensitivity analyses suggested that this did not alter the conclusions with respect to the primary end point of the trial. The current trial did not include an active comparator group, which limits comparison of the results with those of available antidepressants.

In conclusion, in this trial of SAGE-217, an oral, synthetic positive allosteric modulator of $\mathrm{GABA}_{\mathrm{A}}$ receptors, patients who received SAGE-217 had a greater reduction in depressive symptoms at day 15 than patients who received placebo. Larger and longer trials are necessary to determine the durability and safety of SAGE-217 in the treatment of depression.

A data sharing statement provided by the authors is available with the full text of this article at NEJM.org.

Supported by Sage Therapeutics.

Disclosure forms provided by the authors are available with the full text of this article at NEJM.org.

We thank members of the Sage Therapeutics Clinical Operations team, including Amanda Moore, Tressa Gamache, Jasmine Alhassani, and Mollie Baird, M.P.H., for the planning, management, and execution of the clinical trials, and Jeffrey R. Skaar, Ph.D., of Boston Strategic Partners and Claire Sauvageot, Ph.D., of Sage Therapeutics Medical Affairs for their assistance with the writing of the manuscript.

\section{REFERENCES}

1. Rush AJ, Trivedi MH, Wisniewski SR, et al. Bupropion-SR, sertraline, or venlafaxine-XR after failure of SSRIs for depression. N Engl J Med 2006;354:1231-42. 2. Trivedi MH, Rush AJ, Wisniewski SR, et al. Evaluation of outcomes with citalo- pram for depression using measurementbased care in $S T A R^{\star} D$ : implications for clinical practice. Am J Psychiatry 2006; 163:28-40.

3. American Psychiatric Association. Practice guideline for the treatment of pa- tients with major depressive disorder. October 2010 (https://psychiatryonline.org/ pb/assets/raw/sitewide/practice_guidelines/ guidelines/mdd.pdf).

4. Luscher B, Shen Q, Sahir N. The GABAergic deficit hypothesis of major de- 
pressive disorder. Mol Psychiatry 2011;16: 383-406.

5. Gerner RH, Hare TA. CSF GABA in normal subjects and patients with depression, schizophrenia, mania, and anorexia nervosa. Am J Psychiatry 1981;138:1098101.

6. Honig A, Bartlett JR, Bouras N, Bridges PK. Amino acid levels in depression: a preliminary investigation. J Psychiatr Res 1988;22:159-64.

7. Mann JJ, Oquendo MA, Watson KT, et al. Anxiety in major depression and cerebrospinal fluid free gamma-aminobutyric acid. Depress Anxiety 2014;31: 814-21.

8. Gabbay V, Mao X, Klein RG, et al. Anterior cingulate cortex $\gamma$-aminobutyric acid in depressed adolescents: relationship to anhedonia. Arch Gen Psychiatry 2012;69: 139-49.

9. Sanacora G, Gueorguieva R, Epperson $\mathrm{CN}$, et al. Subtype-specific alterations of gamma-aminobutyric acid and glutamate in patients with major depression. Arch Gen Psychiatry 2004;61:705-13.

10. Schür RR, Draisma LW, Wijnen JP, et al. Brain GABA levels across psychiatric disorders: a systematic literature review and meta-analysis of (1) H-MRS studies. Hum Brain Mapp 2016;37:3337-52.

11. Hasler G, van der Veen JW, Tumonis T, Meyers N, Shen J, Drevets WC. Reduced prefrontal glutamate/glutamine and gammaaminobutyric acid levels in major depression determined using proton magnetic resonance spectroscopy. Arch Gen Psychiatry 2007;64:193-200.

12. Guilloux JP, Douillard-Guilloux G, Kota R, et al. Molecular evidence for BDNF- and GABA-related dysfunctions in the amygdala of female subjects with major depression. Mol Psychiatry 2012;17: 1130-42.

13. Karolewicz B, Maciag D, O'Dwyer G Stockmeier CA, Feyissa AM, Rajkowska G. Reduced level of glutamic acid decarboxylase- $67 \mathrm{kDa}$ in the prefrontal cortex in major depression. Int J Neuropsychopharmacol 2010;13:411-20.

14. Rajkowska G, O’Dwyer G, Teleki Z, Stockmeier CA, Miguel-Hidalgo JJ. GABAergic neurons immunoreactive for calcium binding proteins are reduced in the prefrontal cortex in major depression. Neuropsychopharmacology 2007;32:471-82. 15. Merali Z, Du L, Hrdina P, et al. Dysregulation in the suicide brain: mRNA expression of corticotropin-releasing hor- mone receptors and GABA(A) receptor subunits in frontal cortical brain region. J Neurosci 2004;24:1478-85.

16. Paul SM, Purdy RH. Neuroactive steroids. FASEB J 1992;6:2311-22.

17. Rupprecht R, Holsboer F. Neuroactive steroids: mechanisms of action and neuropsychopharmacological perspectives. Trends Neurosci 1999;22:410-6.

18. Rupprecht R, Reul JM, Trapp T, et al. Progesterone receptor-mediated effects of neuroactive steroids. Neuron 1993;11:52330.

19. Zorumski CF, Paul SM, Izumi Y, Covey DF, Mennerick S. Neurosteroids, stress and depression: potential therapeutic opportunities. Neurosci Biobehav Rev 2013; 37:109-22.

20. Uzunov DP, Cooper TB, Costa E, Guidotti A. Fluoxetine-elicited changes in brain neurosteroid content measured by negative ion mass fragmentography. Proc Natl Acad Sci U S A 1996;93:12599-604.

21. Vallée M, Rivera JD, Koob GF, Purdy RH, Fitzgerald RL. Quantification of neurosteroids in rat plasma and brain following swim stress and allopregnanolone administration using negative chemical ionization gas chromatography/mass spectrometry. Anal Biochem 2000;287:153-66.

22. Dong E, Matsumoto K, Uzunova V, et al. Brain 5alpha-dihydroprogesterone and allopregnanolone synthesis in a mouse model of protracted social isolation. Proc Natl Acad Sci U S A 2001;98:2849-54

23. Pibiri F, Nelson M, Guidotti A, Costa E, Pinna G. Decreased corticolimbic allopregnanolone expression during social isolation enhances contextual fear: a model relevant for posttraumatic stress disorder. Proc Natl Acad Sci U S A 2008;105:556772 .

24. Uzunova V, Sheline Y, Davis JM, et al. Increase in the cerebrospinal fluid content of neurosteroids in patients with unipolar major depression who are receiving fluoxetine or fluvoxamine. Proc Natl Acad Sci U S A 1998;95:3239-44.

25. Kanes S, Colquhoun H, Gunduz-Bruce $\mathrm{H}$, et al. Brexanolone (SAGE-547 injection) in post-partum depression: a randomised controlled trial. Lancet 2017;390:480-9. 26. Meltzer-Brody S, Colquhoun H, Riesenberg $\mathrm{R}$, et al. Brexanolone injection in post-partum depression: two multicentre, double-blind, randomised, placebo-controlled, phase 3 trials. Lancet 2018;392: 1058-70.

27. Althaus AL, Belfort GM, Lewis MC, et al. SAGE-217, a next generation neuroactive steroid positive allosteric modulator of synaptic and extra-synaptic GABAA receptors, is active against a rat model of reserpine-induced oral dyskinesia. Presented at the 69th Annual Meeting of the American Academy of Neurology, Boston, April 22-28, 2017. abstract.

28. Robichaud AJ, Doherty JJ, Salituro FG, et al. SAGE-217: a 2nd generation neuroactive steroid. Presented at the 68th Annual Meeting of the American Epilepsy Society, Seattle, December 5-9, 2014. abstract.

29. Bialer M, Johannessen SI, Levy RH, Perucca E, Tomson T, White HS. Progress report on new antiepileptic drugs: a summary of the Twelfth Eilat Conference (EILAT XII). Epilepsy Res 2015;111:85-141. 30. Martinez Botella G, Salituro FG, Harrison BL, et al. Neuroactive steroids. 2 . $3 \alpha$-Hydroxy-3 $\beta$-methyl-21-(4-cyano- $1 \mathrm{H}$ -pyrazol-1'-yl)-19-nor-5 $\beta$-pregnan-20-one (SAGE-217): a clinical next generation neuroactive steroid positive allosteric modulator of the ( $\gamma$-aminobutyric acid $)_{A}$ receptor. J Med Chem 2017;60:7810-9.

31. Kanes S, Nomikos G, Quirk M, et al. A multiple-ascending dose study of the neuroactive steroid SAGE-217. Biol Psychiatry 2017;81:Suppl:S347. abstract.

32. Kanes S, Nomikos G, Quirk M, et al. A single-ascending dose study of the neuroactive steroid SAGE-217. Biol Psychiatry 2017;81:Suppl:S31. abstract.

33. Gunduz-Bruce H, Riesenberg R, Sankoh A, et al. SAGE-217 in subjects with major depressive disorder: efficacy and safety results from open-label Part A of a phase 2A study. Eur Neuropsychopharmacol 2017; 27:S856-S857.

34. Hamilton M. A rating scale for depression. J Neurol Neurosurg Psychiatry 1960;23:56-62.

35. Diagnostic and statistical manual of mental disorders, 5th ed. Washington, DC: American Psychiatric Association, 2013.

36. Bech P, Allerup P, Gram LF, et al. The Hamilton Depression Scale: evaluation of objectivity using logistic models. Acta Psychiatr Scand 1981;63:290-9.

37. Busner J, Targum SD. The Clinical Global Impressions Scale: applying a research tool in clinical practice. Psychiatry (Edgmont) 2007;4:28-37.

38. Luscher B, Fuchs T. GABAergic control of depression-related brain states. Adv Pharmacol 2015;73:97-144.

Copyright $(2019$ Massachusetts Medical Society. 